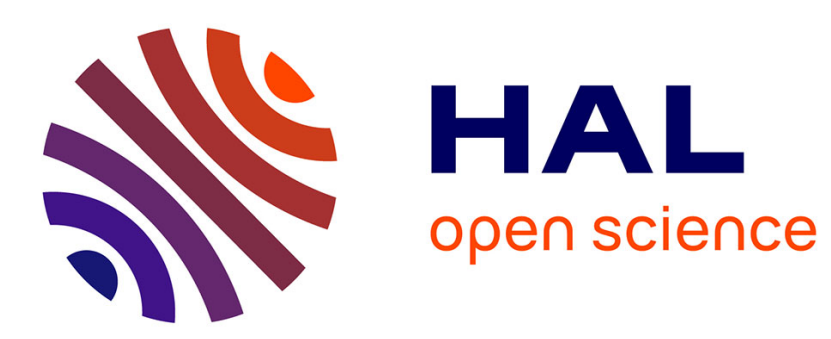

\title{
Dynamic Parameters Optimization and Identification of a Parallel Robot
}

Taha Houda, Ali Amouri, Lotfi Beji, Malik Mallem

\section{To cite this version:}

Taha Houda, Ali Amouri, Lotfi Beji, Malik Mallem. Dynamic Parameters Optimization and Identification of a Parallel Robot. Andrés Kecskeméthy; Francisco Geu Flores. Multibody Dynamics 2019, 53, Springer, pp.367-374, 2019, Computational Methods in Applied Sciences, 978-3-030-23131-6. hal-02180507

\section{HAL Id: hal-02180507 https://hal.science/hal-02180507}

Submitted on 30 Sep 2019

HAL is a multi-disciplinary open access archive for the deposit and dissemination of scientific research documents, whether they are published or not. The documents may come from teaching and research institutions in France or abroad, or from public or private research centers.
L'archive ouverte pluridisciplinaire HAL, est destinée au dépôt et à la diffusion de documents scientifiques de niveau recherche, publiés ou non, émanant des établissements d'enseignement et de recherche français ou étrangers, des laboratoires publics ou privés. 


\title{
Dynamic parameters optimization and identification of a parallel robot
}

\author{
Taha HOUDA, Ali AMOURI, Lotfi BEJI and Malik MALLEM
}

\begin{abstract}
A common issue of the parallel robot is that has a large number of dynamic parameters, which requires a lot of processing time, whether in dynamic modeling, identification or control. The optimization and estimation of inertial parameters of a large DoF number of robotic system is crucial to tune the model-based control law in order to improve the robot accuracy. In this paper, we present an optimized number of dynamic parameters, called base inertial parameters. As a result, only 90 base inertial parameters affect the evr@ simulator instead of 210 standard one. Torque signals evaluation from experimental parallel platform and the developed analytical form show the effectiveness of the obtained results.
\end{abstract}

\section{Introduction}

It is well known that dynamic parameters play an important role in describing the behavior of a multibody system such as robot manipulators [1]. Hence, an optimized analytical form of the dynamic model must be developed, and the number of mathematical operations should be reduced. Thus, a control based on an optimized dynamic model can be build and easily implemented in real time. The Gouph-Stewart platform has been extensively studied in the literature from modeling and control views due to the high stiffness, payload capacity and high accuracy that occur [2] [3]. In addition, the Gough-Stewart platform has retained the attention of industries, and a lot of tests and applications have been developed. One cites, for example, simulators for land vehicles in the automotive industry, flight simulators in the fields of aeronautics and submarine simulators as well as they have been used as manipulators. The Ibisc laboratory, through the IRA2 team, is currently working on the dynamic optimization and control in the mixed reality environment of the Gough-

Taha HOUDA, Ali AMOURI, Lotfi BEJI and Malik MALLEM

IBISC Laboratory, University of Evry / Paris Saclay ,40 Rue du Pelvoux, 91020, Evry

e-mail: (Taha.Houda, Ali.Amouri, Lotfi.Beji, Malik.Mallem)@univ-evry.fr 
Stewart platform. As shown by figure 1, the parallel platform (6-DOF) is fixed on two sliding joints, and where the operator should be attached to the upper platform. This platform was conducted and built by our team.

Consequently, we are dealing with an 8-DOF motion system which is attended to be used as a simulator for people with reduced mobility (disabled person) [4]. The system's objectives are to create a real feeling motion when the human is merged into mixed reality environments. This system will be used in several fields such as rehabilitation for people with motor disabilities, rescue environments and a sport simulator for educational purposes. The combination of the small displacement asserted by the parallel platform and the large displacements of sliders, all of these allow a large operator's workspace for several types of sports, such as ski, wake and jet ski simulations. Consequently, an agreement is necessary such that the upper platform trajectories integrating disabled human objectives are stable (to ensure safety). Hence, we resume our contribution by the following: to improve the controllability, performance and reliability of the parallel simulator, a description of the Evr@ simulator was carried out in section 2, a dynamic optimization of the simulator parameters has been detailed through the energy model in section 3 , followed by a detailed dynamic parameters identification in section 4 .

\section{System Description}

The multi-body structure consists of 6-DoF Gough-Stewart platform mounted on a 2-DoF slider, these two sliders carry both the 6-DoF platform and the axes as shown in the Fig. 1.

As it is known, the GoughStewart platform consists of six legs and two platforms, where the lower platform fixed on the two sliders called the base platform and the other called the upper platform, each leg is connected on one side, to the base platform by a cardan joint and on the other side by a spherical joint, each of the 8 joints is actuated using a hybrid Nanotec PD4 servomotor.

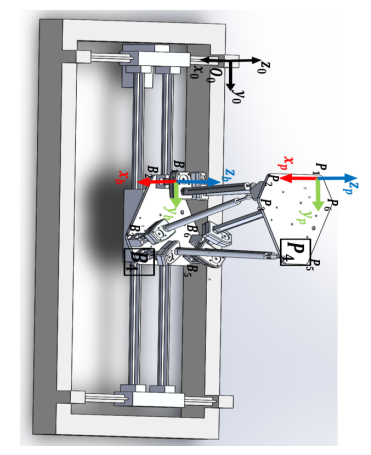

Fig. 1: Evr@ simulator

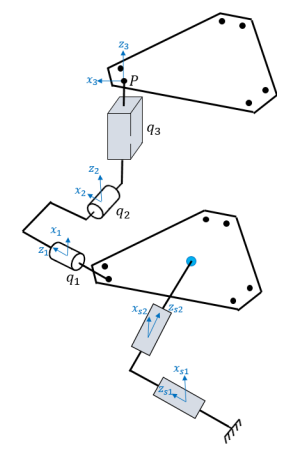

Fig. 2: leg parameterization

The rotational movement of the motors mounted on the leg is transformed to translational movement due to the high-quality ball screw-up system. The 8 brushless motor are controlled by CAN-Open Protocol. The motor has three embedded sensors for position, velocity and torque. The information is collected with a sam- 
pling time of about $0.0196 \mathrm{~s}$, which corresponds to $51 \mathrm{~Hz}$ sampling frequency. All the system has been designed, manufactured and programmed within the IRA2 team.

\section{Dynamic Parameters Optimization}

Such a procedure requires a dynamic based control procedure where all system parameters must to be minimized and identified [5]. With respect to (DenavitHartenberg) parametrization and following notations given by Khalil [6], the dynamic of the studied system depends on 210 dynamic parameters knowns as standard inertial parameters (10 parameters per link, 2 sliding links, 3 links per leg and upper platform link). In the following, we detail the methods carried to optimize the number of dynamic parameters.

Proposition 1 For a given link, in the link energy, if the column of $h_{k}$ is equal to zero or a constant number, the corresponding inertial parameter can be eliminated.

Proof. Let us introduce the total energy $H_{k}$ of the $k^{\text {th }}$ link

$$
H_{k}=E_{k}+U_{k}=\left(e_{k}+u_{k}\right) \xi_{k}^{i n}=h_{k} \xi_{k}^{i n}
$$

where $E_{k}$ is the kinetic energy of the $k^{\text {th }}$ link,

$$
\begin{aligned}
E_{k} & =\frac{1}{2}\left[\omega_{k}^{T} J_{k} \omega_{k}+M_{k} V_{k}^{T} V_{k}+2 M S_{k}^{T}\left(V_{k} \times \omega_{k}\right)\right] \\
& =e_{k} \xi_{k}^{\text {in }}
\end{aligned}
$$

and $U_{k}$ is the potential energy of the $k^{\text {th }}$ link,

$$
\begin{aligned}
U_{k} & =-M_{k}{ }^{0} g^{T}\left({ }^{0} P_{k}+{ }^{0} A_{k}{ }^{k} S_{k}\right) \\
& =u_{k} \xi_{k}^{i n}
\end{aligned}
$$

where $e_{k}$ and $u_{k}$ are functions of the kinematic variables, given in Appendix. The link $k$ standard inertial parameters and $h_{k}$ vector are defined by:

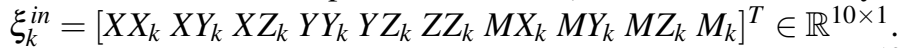

$h_{k}=\left[h_{X X_{k}} h_{X Y_{k}} h_{X Z_{k}} h_{Y Y_{k}} h_{Y Z_{k}} h_{Z Z_{k}} h_{M X_{k}} h_{M Y_{k}} h_{M Z_{k}} h_{M_{k}}\right]^{T} \in \mathbb{R}^{10 \times 1}$.

Therefore, $k^{\text {th }}$ link energy $H_{k}$ is as:

$$
H_{k}=\sum_{j=1}^{r_{2}} h_{k}^{j} \xi_{k}^{j, i n}
$$

where $r_{2}$ is the number of inertial parameters by link, $r_{2}=10, h_{k}^{j}$ is the $j^{\text {th }}$ element of $h_{k}$ vector corresponding to $k^{\text {th }}$ link and $\xi_{i}^{j, i n}$ is the $j^{\text {th }}$ element of the inertial vector corresponding to $i^{\text {th }}$ link.

Let us take the $l^{\text {th }}$ column of link $k h_{k}^{l}=$ const, we can write $H_{k}$ as 


$$
H_{k}=\sum_{j=1}^{l-1} h_{k}^{j} \xi_{k}^{j, i n}+\sum_{j=l+1}^{r_{2}} h_{k}^{j} \xi_{k}^{j, i n}+\text { const }
$$

Since the dynamic effect of such link is seen by the differentiation of the total energy $\Delta H_{k}$, therefore we can set $\xi_{k}^{l, \text { in }}=0$ without changing the value of $\Delta H_{k}$. This means that $\xi_{k}^{l, i n}$ has no effect on the dynamic model, then it can be eliminated.

Proposition 2 For one leg, in the link energy, if the $h_{k}$ column can be expressed as function of the previous link'energy function $h_{k-1}$, then $\xi_{k}^{\text {in }}$ can be grouped with $\xi_{k-1}^{i n}$, and another grouped inertial parameter $\xi_{R, k-1}^{i n}$ appears.

Proof. The robot leg energy $H_{\text {leg }}$ is as:

$$
H_{\text {leg }}=\sum_{i=1}^{r_{1}} \sum_{j=1}^{r_{2}} h_{i}^{j} \xi_{i}^{j, i n}
$$

where $r_{1}$ is the number of link by leg, in Evr@ simulator, $r_{1}=3, h_{i}^{j}$ is the $j^{t h}$ element of the energy vector corresponding to $i^{\text {th }}$ link and $\xi_{i}^{j, i n}$ is the $j^{\text {th }}$ element of the inertial vector corresponding to $i^{\text {th }}$ link, so $h_{i} \xi_{i}^{i n}=\sum_{j=1}^{r_{2}} h_{i}^{j} \xi_{i}^{j, i n}$.

In one leg, let us consider a possible energy linear dependence between $l^{\text {th }}$ column of the link $k$ and link $k-1$, which can take the following form $h_{k}^{l}=h_{k-1} \alpha_{k-1}$, where $\alpha_{k-1} \in \mathbb{R}^{10 \times 1}$. Then,

$$
\begin{aligned}
H_{\text {leg }}= & \sum_{i=1}^{k-2} \sum_{j=1}^{r_{2}} h_{i}^{j} \xi_{i}^{j, i n}+\sum_{i=k+1}^{r_{1}} \sum_{j=1}^{r_{2}} h_{i}^{j} \xi_{i}^{j, i n}+\sum_{j=1}^{r_{2}} h_{k-1}^{j} \xi_{i}^{j, i n}+\sum_{j=1}^{r_{2}} h_{k}^{j} \xi_{i}^{j, i n} \\
= & \sum_{i=1}^{k-2} \sum_{j=1}^{r_{2}} h_{i}^{j} \xi_{i}^{j, i n}+\sum_{i=k+1}^{r_{1}} \sum_{j=1}^{r_{2}} h_{i}^{j} \xi_{i}^{j, i n} \\
& \quad+\sum_{j=1}^{l-1} h_{k}^{j} \xi_{i}^{j, i n}+\sum_{j=l+1}^{r_{2}} h_{k}^{j} \xi_{i}^{j, i n}+h_{k-1} \xi_{k-1}^{i n}+h_{k-1} \alpha_{k-1} \xi_{k}^{l, i n} \\
= & \sum_{i=1}^{k-2} \sum_{j=1}^{r_{2}} h_{i}^{j} \xi_{i}^{j, i n}+\sum_{i=k+1}^{r_{1}} \sum_{j=1}^{r_{2}} h_{i}^{j} \xi_{i}^{j, i n} \\
& \quad+\sum_{j=1}^{l-1} h_{k}^{j} \xi_{i}^{j, i n}+\sum_{j=l+1}^{r_{2}} h_{k}^{j} \xi_{i}^{j, i n}+h_{k-1}\left(\xi_{k-1}^{i n}+\alpha_{k-1} \xi_{k}^{l, i n}\right) \\
= & \sum_{i=1}^{k-2} \sum_{j=1}^{r_{2}} h_{i}^{j} \xi_{i}^{j, i n}+\sum_{i=k+1}^{r_{1}} \sum_{j=1}^{r_{2}} h_{i}^{j} \xi_{i}^{j, i n}+\sum_{j=1}^{l-1} h_{k}^{j} \xi_{i}^{j, i n}+\sum_{j=l+1}^{r_{2}} h_{k}^{j} \xi_{i}^{j, i n}+h_{k-1} \xi_{R, k-1}^{i n}
\end{aligned}
$$

where a grouped parameter can be identified, $\xi_{R, k-1}^{i n}=\xi_{k-1}^{i n}+\alpha_{k-1} \xi_{k}^{l, i n}$

Proposition 3 The 8 DoF optimized dynamics parameters are of number 90 parameters instead of 210 standard inertial parameters 
Proof. At First, let us note that all the link velocities are computed using the recursive Newton-Euler relations such as presented in [6] [7]

$$
\begin{aligned}
{ }^{k} \omega_{k} & ={ }^{k} R_{k-1}{ }^{k-1} \omega_{k-1}+\bar{\sigma}_{k} \dot{q}_{k}{ }^{k} a_{k}={ }^{k} \omega_{k-1}+\sigma_{k} \dot{q}_{k}{ }^{k} a_{k} \\
{ }^{k} V_{k} & ={ }^{k} R_{k-1}\left({ }^{k-1} V_{k-1}+{ }^{k-1} \omega_{k-1} \times{ }^{k-1} P_{k}\right)+\sigma_{k} \dot{q}_{k}{ }^{k} a_{k}
\end{aligned}
$$

All symbols definitions are given in appendix. Form the definition of the kinetic and potential energies, the $h_{k}$ elements can be formulated as given in Table 1.

The columns of $h_{k}$ which are constant:

1. for sliding joint: $h_{X X}, h_{X Y}, h_{X Z}, h_{Y Y}, h_{Y Z}, h_{Z Z}, h_{M X}, h_{M Y}, h_{M Z}$ (9 parameters eliminated)

2. for the first rotational link of each leg: $h_{X X}, h_{X Y}, h_{X Z}, h_{Y Y}, h_{Y Z}$ (5 parameters eliminated by leg)

Now as a direct application of Propostion 1 , a $48(9 \times 2+5 \times 6)$ standard inertial parameters are eliminated. For example, the first element of $h_{k}$ vector of the sliding joint is equal to $h_{s}^{1}=\frac{1}{2} \omega_{1, s} \omega_{1, s}$, as the sliding joint has no rotational movement which means $\omega_{1, s}=0$. Consequently, $h_{s}^{1}=0$ then the $X X_{s}$ parameter is eliminated.

For one leg, the $h_{k}$ columns which have linear dependence with the $h_{k-1}$ previous link, are for the first rotational link: $h_{M Z}, h_{M}$, for the second rotational link: $h_{Y Y} h_{M Z}, h_{M}$ and for the prismatic link: $h_{X X}, h_{X Y}, h_{X Z}, h_{Y Y}, h_{Y Z}, h_{Z Z}, h_{M}$. After application of Proposition 2, 72 standard inertial parameters are grouped, 12 by leg. Example, for one leg (according to the notation of the Fig. 1 and Fig. 2),

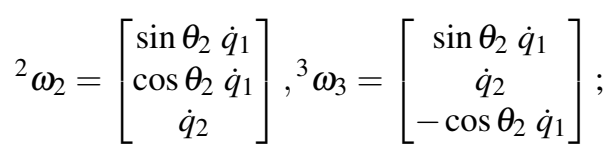

we can find that $h_{3}^{1}=\left(\cos \theta_{2} \dot{q}_{1}\right)^{2}, h_{2}^{4}=\left(\cos \theta_{2} \dot{q}_{1}\right)^{2}$ which gives us $h_{3}^{1}=h_{2} \alpha_{2}$ where $\alpha_{2}=\left[\begin{array}{llllllllll}0 & 0 & 0 & 1 & 0 & 0 & 0 & 0 & 0 & 0\end{array}\right]^{T}$. Consequently, The $X X_{3}$ inertial parameter is grouped with link-2 inertial parameters $\xi_{R, 2}^{i n}=\xi_{2}^{i n}+\alpha_{2} \xi_{3}^{l, i n}$ following the equation (9).

\section{Dynamic parameters identification}

An energy model based on the optimized dynamic parameters has been applied in the following form, so we can write:

$$
\begin{aligned}
\Delta H & =T^{T} d q \\
& =T^{T} \dot{q} d t
\end{aligned}
$$

where,

$$
T=\Gamma-\operatorname{diag}(\operatorname{sign}(\dot{q})) F_{s}-\operatorname{diag}(\dot{q}) F_{v}
$$


After integration,

$$
\int_{t_{1}}^{t_{2}} T^{T} \dot{q} d t=H\left(t_{2}\right)-H\left(t_{1}\right),
$$

Hence, the total energy can be expressed linearly as function of the inertial and friction parameters, we can write:

$$
\begin{aligned}
\mathbf{Y}=\int_{t_{1}}^{t_{2}} \Gamma^{T} \dot{q} d t & =\Delta H+\Delta H_{F_{v}}+\Delta H_{F_{s}} \\
& =\Delta h \xi^{i n}+\Delta h_{F_{v}} F_{v}+\Delta h_{F_{s}} F_{s}
\end{aligned}
$$

Therefore, the identification energy model form is as follow,

$$
\begin{gathered}
\mathbf{Y}=\int_{t_{1}}^{t_{2}} \Gamma^{T} \dot{q} d t=\left[\Delta h \Delta h_{F_{v}} \Delta h_{F_{s}}\right]\left[\begin{array}{l}
\xi^{i n} \\
F_{v} \\
F_{S}
\end{array}\right] \\
\mathbf{Y}(\Gamma, \dot{q})=W(q, \dot{q})\left[\begin{array}{l}
\xi^{i n} \\
F_{v} \\
F_{s}
\end{array}\right]+\rho
\end{gathered}
$$

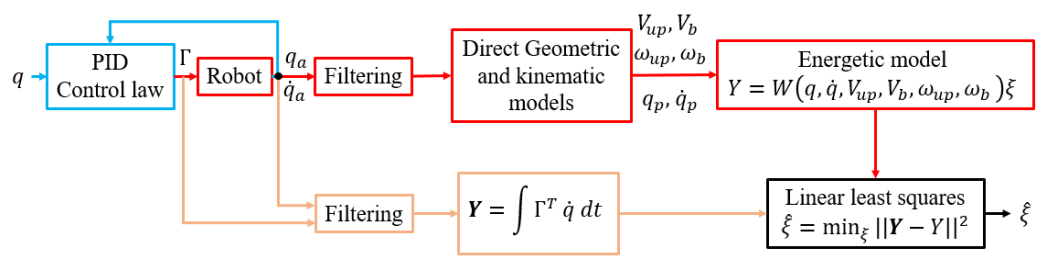

Fig. 3: Schematic diagram of the energy model.

$\Gamma \in \mathbb{R}^{8}$ is the vector of generalized torques, $q=\left[q_{a}, q_{p}\right], \dot{q}=\left[\dot{q}_{a}, \dot{q}_{p}\right]$ where $q_{a} \in$ $\mathbb{R}^{8}, q_{p} \in \mathbb{R}^{12}$ are the active and passive joint positions, $\left(V_{b} \times \omega_{b}\right)$ and $\left(V_{u p} \times \omega_{u p}\right) \in$ $\left(\mathbb{R}^{3} \times \mathbb{R}^{3}\right)$ are the speed vectors of the base and the upper platform respectively, $\xi=\left[\xi^{i n}, \xi^{f}\right]$ where $\xi^{i n}, \xi^{f}=\left[F_{v}, F_{s}\right]^{T}$ are the inertial and friction parameters respectively, $F_{v}, F_{S}$ are the viscous and dry friction coefficients.

Several exciting trajectories has been applied with the highest velocity to take around 50000 equations ( $>500 \times$ number of parameters) to make sure that the trajectories have enough information, all detected variables such as displacements, velocities and torques were filtered in order to eliminate the high frequency which is harmful for the identification procedure.

A least square method has been used to solve this overdetermined system

$$
\hat{\xi}=\left[W^{T} W\right]^{-1} W^{T} \mathbf{Y}
$$


the precision of the identified parameter $\hat{\xi}$ is depend on the persistence of the observation matrix [8] [9].

The identification results of two sliding joints are shown in the Table 1.

Table 1: Two sliding joints inertial parameters

\begin{tabular}{llllll}
\hline Axes & $\mathrm{M}(\mathrm{Kg})$ & $F_{v_{1}}(N . s / \mathrm{mm})$ & $F_{s_{1}}(N)$ & $F_{v_{2}}(N . s / m m)$ & $F_{s_{2}}(N)$ \\
\hline First & 33,7 & 76,38 & 9,14 & 72,38 & 11.23 \\
Second & 20,56 & 37,30 & 9,60 & 42,98 & 10,44 \\
\hline
\end{tabular}

$F_{v_{1}}, F_{v_{2}}, F_{s_{1}}, F_{s_{2}}$ Viscous and sec frictions in the first and second directions respectively.

In order to verify the identification results, a torque construction from the model using the Lagrangian formulation and the identified parameters is performed. The Lagrangian is equal to the total kinetic energy differentiated by the total potential energy:

$L=E-U=e_{k} \hat{\xi}_{k}^{i n}-u_{k} \hat{\xi}_{k}^{i n} ; \quad \Gamma_{\text {Model }}=\Gamma_{\hat{\xi}^{i n}}+\Gamma_{\hat{\xi}^{f}}$

An example of torque detected and constructed of leg $B_{4} P_{4}$ (Fig. 1) is shown beside.

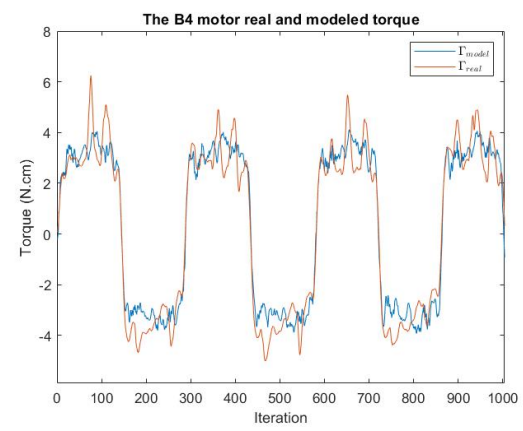

The $B_{4} P_{4}$ axis friction was taken in two directions to take into account the dissymmetry of the friction model, we note 1 and 2 for the first and the second direction, respectively: $F_{s_{1}}=1,39 \mathrm{~N}, F_{v_{1}}=56,98 \mathrm{~N} . \mathrm{s} / \mathrm{mm}, F_{s_{2}}=1,33 \mathrm{~N}, F_{v_{2}}=58,31 \mathrm{~N} . \mathrm{s} / \mathrm{mm}$, Finally, the grouped mass of upper platform and last 6 links is $M_{R_{p}}=6.15 \mathrm{Kg}$.

\section{Conclusion and Perspective}

The results of this work benefit from an adequate parallel robot's parametrization studied in [6]. Consequently, the 210 standard inertial parameters are reduced to 90 base inertial parameters only. The given procedure has led to an optimized dynamic/energetic model where the implementation in real time is possible. Obtained torques, experimentally sensed and analytically computed, are comparable. The next steps and perspectives will necessarily focus in the multi-body system control using the identified dynamic parameters and study of the hemiplegic skier's reaction and his rehabilitation enhancement in immersion environment. 


\section{Appendix}

Table 2: $e_{k}$ and $u_{k}$ vector elements

\begin{tabular}{|c|c|c|c|c|c|}
\hline$\xi_{k}^{i n}$ & $X X$ & $X Y$ & $\mathrm{XZ}$ & YY & $\mathrm{YZ}$ \\
\hline $\begin{array}{l}e_{k} \\
u_{k}\end{array}$ & $\begin{array}{l}\frac{1}{2} \omega_{1, k} \omega_{1, k} \\
0\end{array}$ & $\frac{1}{2} \omega_{1, k} \omega_{2, k}$ & $\begin{array}{l}\frac{1}{2} \omega_{1, k} \omega_{3, k} \\
0\end{array}$ & $\frac{1}{2} \omega_{2, k} \omega_{2, k}$ & $\frac{1}{2} \omega_{2, k} \omega_{3, k}$ \\
\hline$\xi_{k}^{i n}$ & $\mathrm{ZZ}$ & MX & MY & $\mathrm{MZ}$ & M \\
\hline$e_{k}$ & $\frac{1}{2} \omega_{3, k} \omega_{3, k}$ & $\omega_{3, k} V_{2, k}-\omega_{2, k} V_{3, k}$ & $\omega_{1, k} V_{3, k}-\omega_{3, k} V_{1, k}$ & $\omega_{2, k} V_{1, k}-\omega_{1, k} V_{2, k}$ & $\frac{1}{2}{ }^{k} V_{k}^{T k} V_{k}$ \\
\hline$u_{k}$ & 0 & $-{ }^{0} g^{T 0} s_{k}$ & $-{ }^{0} g^{T 0} n_{k}$ & $-{ }^{0} g^{T 0} a_{k}$ & $-{ }^{0} g^{T 0} P_{k}$ \\
\hline
\end{tabular}

Where, ${ }^{k} \omega_{k}=\left[\begin{array}{lll}\omega_{1, k} & \omega_{2, k} & \omega_{3, k}\end{array}\right]^{T},{ }^{k} V_{k}=\left[\begin{array}{lll}V_{1, k} & V_{2, k} & V_{3, k}\end{array}\right]^{T}$ and ${ }^{0} R_{k}$ and ${ }^{0} P_{k}$ are the matrix and vector appearing in the transformation matrix ${ }^{0} T_{k}$ with the rotation matrix ${ }^{0} R_{k}=\left[{ }^{0} s_{k}{ }^{0} n_{k}{ }^{0} a_{k}\right], a_{k}$ unit vector along $Z_{k}$ direction, ${ }^{k} J_{k}-(3 \times 3)$ inertia tensor of link $k$ with respect to frame $F_{k} . M_{k}$ and $M S_{k}$ are the mass and first moment of inertia of link $k$ respectively.

\section{References}

1. Wisama Khalil, Sylvain Guegan. Inverse and Direct Dynamic Modeling of Gough-Stewart Robots. IEEE Transactions on Robotics and Automation, Institute of Electrical and Electronics Engineers (IEEE), 2004.

2. Merlet J.-P., Parallel robots, Dordrecht, The Netherland, Kluwer, 2000.

3. Harib, Khalifa, Srinivasan, Krishnaswamy. (2003). Kinematic and dynamic analysis of Stewart platform-based machine tool structures. Robotica.

4. Ali Amouri, Fakhr-Eddine Ababsa. Sliding Movement Platform for Mixed Reality Application. 7th IFAC Symposium on Mechatronic Systems, Sep 2016, Loughborough, United Kingdom. 49 (21), pp.662-667

5. Wisama Khalil, Maxime Gautier, Philippe Lemoine. Identification of the payload inertial parameters of industrial manipulators. IEEE International Conference on Robotics and Automation - ICRA'07, Apr 2007, Rome, Italy. pp. 4943-4948, 2007

6. Khalil W., Dombre E., "Modeling, identification and control of robots", Hermès Penton, London, 2002.

7. Luh J.Y.S., Walker M.W., Paul R.C.P., "On-line computational scheme for mechanical manipulators", Trans. of ASME, J. of Dynamic Systems, Measurement, and Control, Vol. 102(2), pp. 69-76, 1980.

8. Kozłowski, Krzysztof. (1996). Experimental identification of dynamic parameters for a class of geared robots. Robotica. 14. 561 - 574.

9. P. O. Vandanjon, M. Gautier and P. Desbats, "Identification of robots inertial parameters by means of spectrum analysis," Proceedings of 1995 IEEE International Conference on Robotics and Automation, Nagoya, Japan, 1995, pp. doi: 10.1109 